\title{
Reviewer Acknowledgements for Vol. 6, No. 3, September 2018
}

Journal of Agricultural Studies would like to acknowledge the following reviewers for their assistance with peer review of manuscripts for this issue. Many authors, regardless of whether JAS publishes their work, appreciate the helpful feedback provided by the reviewers. Their comments and suggestions were of great help to the authors in improving the quality of their papers. Each of the reviewers listed below returned at least one review for this issue.

\section{Reviewers for Volume 6, Number 3}

Abhishek A. Cukkemane, Bijasu Agri Research Laboratory LLP, India

Anil Kumar Matta, Vaddeswaram, Guntur dst, India

Babak Mohammadi, University of Tehran, Iran

Chenlin Hu, The Ohio State University, USA

Eben von Well, Agricultural Research Council, South Africa

Eliana Mariela Werbin, National University of Cordoba, Argentinian

Ernest Baafi, CSIR-Crops Research Institute, Ghana

Ewa Moliszewska, Opole University, Poland

Gerardo Ojeda, Universidad Nacional de Colombia, Colombia

Pramod Kumar Mishra, University of Hyderabad, India

Sahar Bahmani, University of Wisconsin at Parkside, USA

Sait Engindeniz, Ege University Faculty of Agriculture, Turkey

Zakaria Fouad Abdallah, National Research Centre, Egypt

Zhao Chen, Clemson University, USA

Zoi M. Parissi, School of Forestry and Natural Environment Aristotle University, Greece

Richard Williams,

Editor

Journal of Agricultural Studies

Macrothink Institute

5348 Vegas Dr.\#825

Las Vegas, Nevada 89108

United States

Phone: 1-702-953-1852 ext.521

Fax: 1-702-420-2900

Email: jas@macrothink.org

URL: http://jas.macrothink.org 